\title{
External Environmental Impact for Evolutionary Prisoner's Games on Lattices
}

\author{
Wanting Gao and Qiqing Song* \\ College of Science, Guilin University of Technology, Guilin 541004, China \\ ${ }^{*}$ Corresponding author
}

\begin{abstract}
This paper studies the role of external environment in the evolution of cooperation. Due to the bounded rationality of players, external environment of individuals does matters on the evolution. The more the amount of cooperators a player's neighborhood has, expectantly, the more confident the player will be in its current choice. Based on this point, two updating rules on regular lattices are introduced concerning external environmental effects. For the first rule, a focal player's payoff is affected proportionally with the difference between the number of cooperators and that of defectors in its group; in the second case, one's payoff is exponentially effected by that difference in its neighbors. On account of the external influence to central individuals, simulation results show that the external environment exerts a significant positive function on the increasing of cooperation level in populations.
\end{abstract}

Keywords-external environment; cooperation; prisoner's dilemma; evolutionary games

\section{INTRODUCTION}

Darwin pointed out that there is no instinct creature for the benefit of others in his book "The Origin of Species" . In other words, all animals oriented by maximizing self-benefits manifest strong selfishness in nature. According to Darwin's theory, the essence of evolution is natural competition, then natural selection leads to the survival of the fittest. It is a widespread field where populations comprise of egocentric individuals, however, cooperation phenomena are observed frequently in different kinds of communities. This gives rise to many studies for the production and maintenance of cooperation. In fact, cooperation became the third principle of natural evolution out of the other two, gene mutation and natural selection [1]. In the study of evolution of cooperation[2,3], one of the most typical models is the prisoner's dilemma based on its conciseness in form and profoundness in reflecting the contradiction between individual rationality and collective rationality [4-6].

By employing the grid network space, the number of cooperators increased because of net reciprocity [7]. After the seminal paper, a large amount of works are devoted to exploring factors of promoting cooperation on lattices, such as, punishment and reward [8-10], reputation[11,12], weight[13,14], age structure [23], and others (see [16-22]), etc.

It should be pointed out that, in all above references, the total payoff of a player is routinely calculated by the sum of the payoffs caused by the pairwise games in relation to all its neighbors. That is to say, all neighbors of a player choosing cooperation will have no any effect on the confidence of its own current status, similarly, it also has no positive or negative influence on the belief of the player's own strategy if all its neighbors are defectors. Therefore, this paper will attach importance to the effects from external environment on the evolution of cooperation.

The paper is organized as follows: firstly, in Section 2 it introduces evolving prisoner's dilemma game models in detail with linear and exponential external environment. Secondly, we are going to present results by simulation so as to account for the behaviors of the evolving models in Section 3. Finally, we give the discussion with some concluding remarks.

\section{The EVOlutionary GAME MODEL WITH EXTERNAL ENVIRONMENT ON LATTICES}

On a square lattice with periodic boundaries condition, every node is occupied by a player. Every player will play a prisoner's dilemma (PD) game with each one of its four nearest neighbors. For a game pair, that both select cooperation results in the fact that each will obtain a reward $R$; if one player chooses to be a cooperator, the other decides to defect, then, the defector gets a temptation $T$, with $T>R$, while the sucker's payoff is $S$ with $S<R$. If two defectors meet, they are punished with a payoff $P$ which is lower than $R$. In general, we have the relation among these payoffs such that $T>R>P>S$.

For the initial status, each player is arranged as either a cooperator $(C)$ or a defector $(D)$ with equal probability. Denote the strategies of a cooperator $x$ and a defector $y$ by

$$
S_{X}=(1,0)^{T}
$$

and

$$
S_{y}=(0,1)^{T}
$$

respectively.

Specifically, in whole paper, we set the payoffs as: $R=1$, $P=S=0, T=b(1<b<2)$, like the typical setting in the paper [15]. At every time step, a player $x$ gets the payoff $p^{x}$ by playing the games with all its neighbors, that is,

$$
p^{X}=\sum_{\mathrm{y} \in \Omega^{X}}{ }^{X} s_{X}^{T} A s_{y}
$$


where $\Omega^{X}$ denotes the four nearest neighbors of $x$. Considering the effect for the player $x$ from its external environment, the player's payoff will be multiplied by a parameter $1+\varepsilon^{X}$ to represent the degree of the influence, where

$$
\varepsilon^{x}=\eta \times \Lambda^{x}
$$

and $\eta$ is a random number in the interval [0,1] and $\Lambda^{X}$ reflects the different situation of the neighbor's strategies; $\Lambda^{x}>0\left(\Lambda^{x}<0\right)$ means that the number of cooperators in the neighborhood of $x$ is more (less) than that of defectors. To be precise, let $n(C)(n(D))$ denote the number of cooperators (defectors) in the player $x$ 's neighbors, subsequently, two different modes related to the external status are considered as follows.

(1) Linear Model: $\Lambda_{\operatorname{lin}}^{X}=\frac{n(C)-n(D)}{n\left(\Omega^{X}\right)}$,

(2) Exponent Model: $\Lambda_{\mathrm{exp}}^{X}=E(n(C)-n(D))_{-1}$,

where is a positive constant; $n\left(\Omega^{x}\right)$ denotes the total number of neighbors of the player $x$, in fact, in the current setting on the lattice, we know that $n\left(\Omega^{X}\right)$ is also a constant such that $n\left(\Omega^{X}\right)=4$.
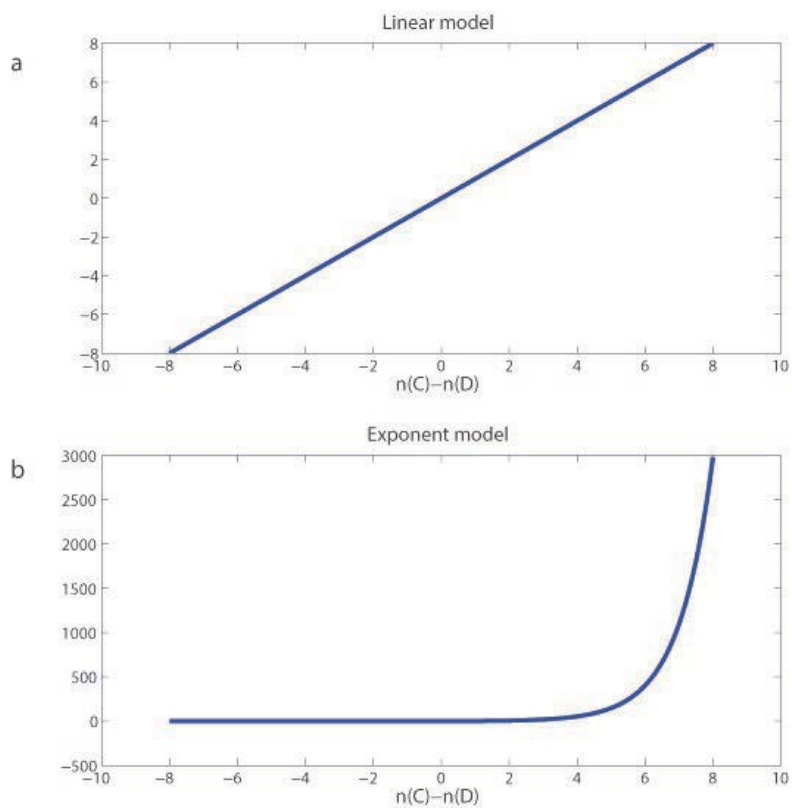

FIGURE I. (A) AND (B) SHOW THE EFFECTS OF LINEAR MODEL

AND EXPONENT MODEL BY DEPICTING $\Lambda^{X}$ WITH THE CHANGE OF $n(C)-n(D)$, RESPECTIVELY; (B) IS A CASE BY USING THE PARAMETER $E$ WITH $E=\mathrm{e}$.
As it is shown, Figure. 1 is generated by the difference between the quantities of cooperators and defectors in one's neighborhood. For the above two models, provided that $n_{C}=n_{D}$, there is no external effect for a focal player's payoff. Figure. 1(b) shows the magnitude of effects by the external environment when $n_{C}>n_{D}$ is more than that when $n_{C}<n_{D}$, which has a different property from the linear case showed in Figure. 1(a) . When the majority are cooperators in the neighbors of a player, the effect is positive; furthermore, whether a player is a cooperator or not, the more cooperators the player's neighborhood has, the more positive effect the player obtains.

In accordance with the above setting, the fitness of a player $x$ is

$$
U_{X}=P_{X}\left(1+\varepsilon_{X}\right)
$$

After one game round, the player $x$ decides the strategy using in the next step, and he/she will follow the strategy of a randomly selected neighbor $y$ with the probability

$$
\omega=\frac{1}{1+\exp \left(\left[U_{x}-U_{y}\right] / K\right)}
$$

where $K$ is uncertainly of selection [25]. In the following part, we set the value $K$ as 0.1 .

In the paper [26], $\Lambda^{X}$ in $\varepsilon_{X}$ is a random number in [-1,1], which means the effect of external environments for the player $x$ is random despite whether the neighbors' cooperation aspiration is strong or not. Whereas, the current setting of $\Lambda^{X}$ is different from that in the paper [26]; and it is clearly that, for this setting of $\Lambda^{X}$ with the strategy updating rule, the higher the number of cooperators one's neighborhood has, the more confidence the player has in its current choice.

\section{The Results OF MONTE CARLO'S Simulations}

We simulate the two models in Section 2 with the different parameter b varying from 1.1 to 1.9 . All individuals simultaneously update their strategy with Eq. (6); as the iteration moves forward, we record the frequencies of different types of players by averaging 50 times initialization.

First of all, under different mechanisms, we observe the change of the level of cooperation with the increase of time steps for different temptation parameters. In order to compare the different mechanisms, Figure. 2(a) shows the case without external effects, that is, the fitness of a player equals its payoff; with the increasing of the temptation value $b$, the level of cooperation in populations decreased significantly. In fact, when $b=1.9$, the cooperation level remains at around 0.5 in all cases, and the whole population consists of an equal amount of cooperators and defectors at last, like Nowak's discovery [24] with the best neighbor imitation rule. In Figure. 2(b), the lines denote the linear model with different parameters $b$, which are in contrast with the cases of Figure .2 (a), and for the case with $b=1.9$, we can find that defectors will quickly disappear, the cooperators in populations take over almost all nodes as the 
time increasing.
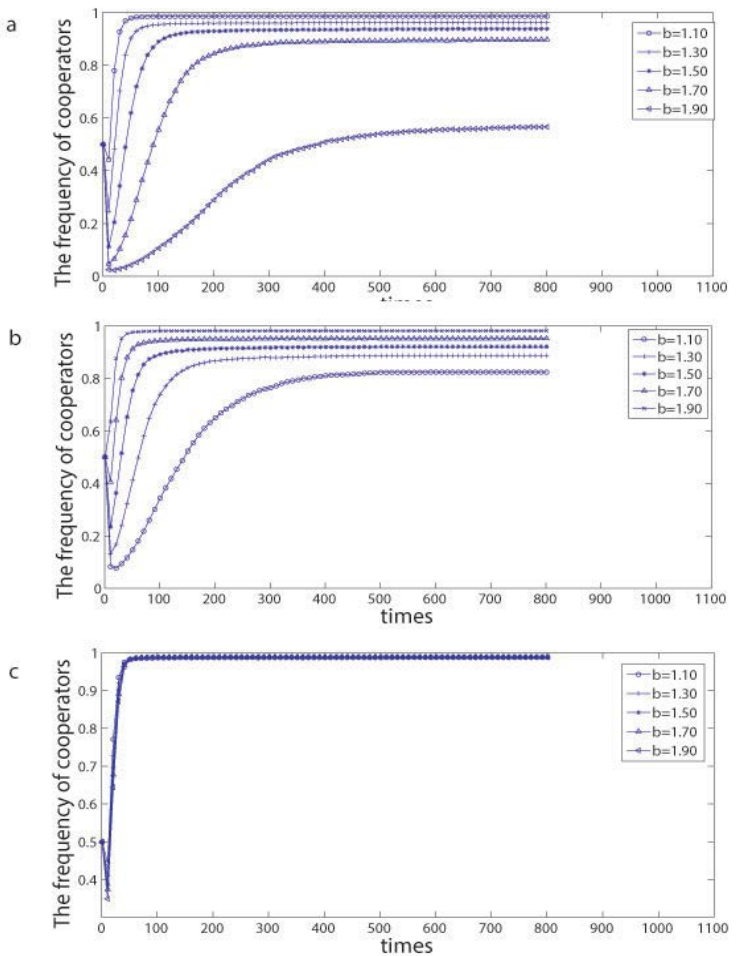

FIGURE II. FRACTION OF COOPERATORS AS A FUNCTION OF TIME STEP FOR DIFFERENT TEMPTATION VALUES B FROM 1.1 TO 1.9. (A) DESCRIBES THE CASE WITHOUT EXTERNAL EFFECTS; (B) IS DRAWN FOR THE LINEAR MODEL; (C) SHOWS THE EXPONENT MODEL WITH E=E. THE LATTICE SIZE M IS 50, AND THE UNCERTAINTY OF STRATEGY ADOPTION $K$ IS 0.1 .
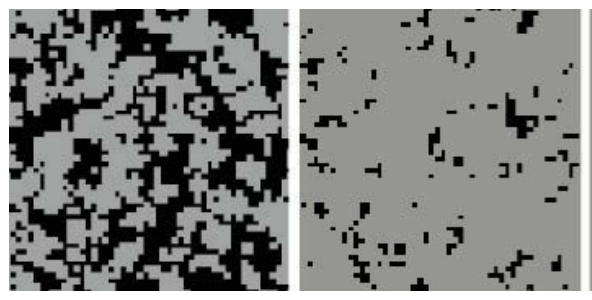

FIGURE III. EVOLUTIONARY SNAPSHOTS FOR THE LINEAR MODEL WITH $b=1.05, \mathrm{~K}=0.1$. FROM LEFT TO RIGHT, THE TIME STEPS ARE 10, 20 AND 100, RESPECTIVELY. COOPERATORS AND DEFECTORS ARE COLORED GRAY AND BLACK RESPECTIVELY.
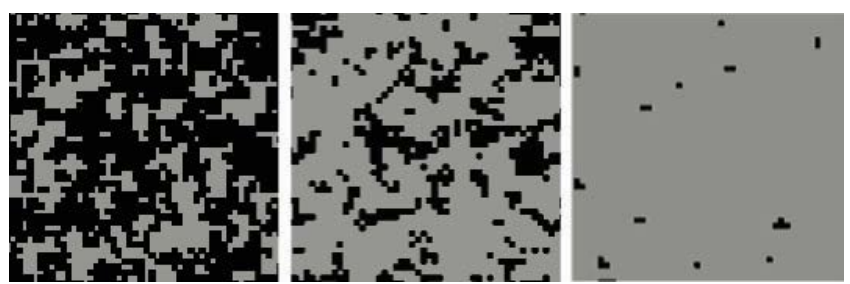

FIGURE IV. EVOLUTIONARY SNAPSHOTS FOR THE EXPONENT MODEL WITH $b=1.05, K=0.1$ AND $E=e$. FROM LEFT TO RIGHT FIGURE, THE TIME STEPS ARE 10, 20 AND 100, RESPECTIVELY. COOPERATORS AND DEFECTORS ARE COLORED GRAY AND BLACK RESPECTIVELY.

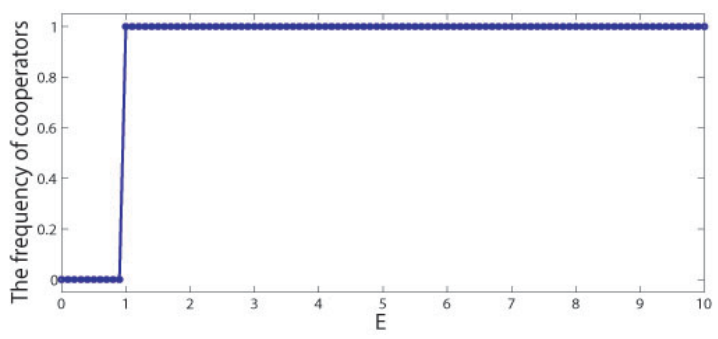

FIGURE V. FRACTION OF COOPERATORS IN STABLE STATUS AS A FUNCTION OF THE PARAMETER $E$. THE PICTURE SHOWS THE RESULTS OF EVOLUTION OF DIFFERENT PARAMETERS IN THE EXPONENT MODEL. ALL THE RESULTS ARE OBTAINED WITH $b=1.05, K=0.1$.

Furthermore, the magnitude of cooperators remarkably enlarged from initial states for all the values of $b$. It should be pointed out that, for the linear model, when the cooperators of a player's neighborhood hold a majority, the player has a positive impact on its payoff, and on the contrary, the player experiences a negative circumstance. Averagely, although it seems that the linear model leads to that the expected value of $\Lambda^{X}$ is zero, the external circumstance effects really produce positive results as it is shown in Figure. 2(b). In Figure. 2(c), the lines represent the case of exponent model with different values of $b$, regardless of the size of $b$, all the frequencies of cooperation increase eventually near to $100 \%$. This shows that the positive impact of the external environment is greater than the negative, and shows that the external environment like the exponent model would be apt to improve the level of cooperation of the population. Figure. 2(b) and Figure. 2(c) reveal that when we are considering of the external influences, both the linear model and the exponent model contribute effectively to enhance the number of the cooperators in groups.

Figure. 3 and Figure. 4 show quite a few snapshots for the evolution of games with the linear model and the exponential model, respectively. When the temptation $b$ is small, the detail of the early stages of the evolutionary process is different between the linear model and the exponent model. The amount of cooperators for the linear model in the early stages is more than that for the exponential case, which demonstrates the diversity of evolution, though, with time going, these cooperators' domains gradually merge and become the only dominated in the stable states.

For these two models on the lattice, if a player is surrounded by two cooperators and two defectors, the player's fitness is just its payoff. Provided that the player is a cooperator, the node will be easily replaced by a defector. Nonetheless, the macroscopic result tells us the external environment plays a significant effect, even for the tiny difference between the number of cooperators and that of defectors in one's neighbors, which makes many defectors replaced by cooperators.

Finally, Figure. 5 dipicts how the level of the cooperation affected by the external environment under these the mechanism when changing the parameter of the exponent model. The line in Figure. 5 implies if the factor $E$ satisfies that $0<E<1$, the level of cooperation in the population is rapidly declined under the influence of the external 
environment; while the factor $E>1$, the cooperators will occupy the whole population. This result illustrates the exponent model can improve the cooperative level of the population within a large range. The threshold of $E$ with $E=1$ shows the critical state whether the population comprising of defectors or cooperators in steady status.

\section{SUMMARY}

We introduce two new models of evolutionary prisoner's games, taking into account that each player in a group is affected by its external environment. In a grid, each individual will be influenced by its neighbors in the processing of prisoner's dilemma games. Then, the fitness of a player depends both on the payoff and its external circumstance.

In concern of the diversity of external environment, two types of effects are considered: the linear or the exponent case, which is in dependence of the quantity difference between cooperators and defectors in the neighborhood of a player. The models remarkably improve the percentage of cooperation in populations, which is very different from the case without the considerations of external environments. For the linear model, there is no defector in the population with $b$ within a certain range. In the exponent case, cooperators will quickly occupy the population even when the temptation parameter is in a large range. The evolution snapshots of the linear model and the exponent model illustrate their game processing: the cooperators will form clusters against the defectors as shown in many references, however, the details in the early stages between these models are different. Finally, we discuss the evolution of cooperation under different parameters of the exponential model: the critical threshold for whether it improves the cooperation level or not is found.

Cooperation is of great significance for the development of nature and society. The contribution of this paper is to illustrate the external circumstance can produce cooperation in populations by simulating the environment which might happen in real life.

\section{ACKNOWLEDGMENT}

This project is supported by National Natural Science Foundation of China (11661030) and Guangxi Natural Science Foundation (2016GXNSFAA380059).

\section{REFERENCES}

[1] M. A. Nowak, "SuperCooperators : altruism, evolution, and why we need each other to succeed,” J. Soc. Polit. Econ. St., vol. 37, 2008, p. 18.

[2] J. M. Smith. The theory of games and the evolution of animal conflicts, Neurosecretion and the biology of neuropeptides. Japan Scientific Societies Press, 1974.

[3] J. M. Smith, G. R. Price, "The Logic of Animal Conflict," Nature, vol. 246, 1973, pp. 15-18.

[4] E. Fehr, U. Fischbacher, "The nature of human altruism: proximate patterns and evolutionary origins," Nature, vol. 425, 2003, pp. 785-791.

[5] J. Hofbauer, K. Sigmund, Evolutionary games and population dynamics. Cambridge University Press, 1998.

[6] M. Milinski, J.H. Lüthi, R. Eggler, et al, "Cooperation under predation risk: experiments on costs and benefits," P. Roy. Soc. Lond. B. Bio., vol. 264, 1997, pp. 831-837.

[7] M. A. Nowak, R. M. May, "Evolutionary games and spatial chaos," Nature, vol. 359, 1992, pp. 826-829.
[8] R. Jimenez, H. Lugo, J. Cuesta, et al, "Emergence and resilience of cooperation in the spatial prisoner's dilemma via a reward mechanism,” J. Theor. Biol., vol. 250, 2008, pp. 475-483.

[9] M. Perc, A. Szolnoki, "Self-organization of punishment in structured populationsNew,” J. Phys., vol. 14, 2012, pp. 43013-43025.

[10] Q. Jin, Z. Wang, Z. Wang, et al, "Strategy changing penalty promotes cooperation in spatial prisoner's dilemma game," Chaos Soliton Fract., vol. 45, 2012, pp. 395-401.

[11] M. Milinski, D. Semmann, H. J. Krambeck, "Reputation helps solve the 'tragedy of the commons'," Nature vol. 415,2002, pp. 424-426.

[12] W. Zhen, W. Lin, Z. Y. Yin, et al, "Inferring Reputation Promotes the Evolution of Cooperation in Spatial Social Dilemma Games," Plos One, vol.7, 2012, p. e40218.

[13] C. Y. Xia, Z. Q. Ma, Y. L. Wang, et al, "Enhancement of cooperation in prisoner's dilemma game on weighted lattices,” Physica A, vol. 390, 2011, pp. 4602-4609.

[14] Z. Q. Ma, C. Y. Xia, S. W. Sun, et al, "Heterogeneous link weight promotes the coopeartion in spatial prisoner's dillemma," Int. J. Mod. Phys., Vol. C22, 2011, pp. 1257-1268.

[15] M. A. Nowak, R. M. May, “The spatial dilemmas of evolution,” Int. J. Bifurcat. Chaos, vol. 3, 1993, pp. 35-78.

[16] M. Perc, Z. Wang, "Heterogeneous aspirations promote cooperation in the prisoner's dilemma game," Plos One, vol. 5, 2010, p. e15117.

[17] D. Helbing, W. Yu. P. "From the Cover: The outbreak of cooperation among success-driven individuals under noisy conditions," Natl. Acad. Sci. USA, vol. 106, 2009, pp. 3680-3685.

[18] M. H. Vainstein, A. T. C. Silva, J. J. Arenzon, "Does mobility decrease cooperation?,” Theor. Biol., vol. 244, 2007, pp. 722-728.

[19] E. Sicardi, H. M. Fort, J. Arenzon. J. "Random mobility and spatial structure often enhance cooperation,” Theor. Biol., vol. 256, 2012, pp. 240-246.

[20] S. Meloni, A. Buscarino, L. Fortuna, et al, "Effects of mobility in a population of prisoner's dilemma players,” Phys. Rev. E, vol. 79, 2009, p. 067101.

[21] Z. Rong, Z. Wu, "Effect of the degree correlation in public goods game on scale-free networks,” Europhys Lett., vol. 87, 2009, p. 30001.

[22] H. Lin, D. P. Yang, J. W. Shuai, "Cooperation among mobile individuals with payoff expectations in the spatial prisoner's dilemma game," Chaos Soliton Fract., vol. 44, 2011, pp. 153-159.

[23] Z. Wang, Z. Wang, X. Zhu, et al, "Cooperation and age structure in spatial gamesPhys,” Rev. E, vol. 85, 2012, p. 011149.

[24] M.A. Nowak, Evolutionary dynamics: exploring the equations of life. Best Seller, 2006.

[25] J. Tanimoto, M. Brede, A. Yamauchi, "Network reciprocity by coexisting learning and teaching strategies," Phys. Rev. E, vol. 85, 2012, p. 032101.

[26] C. Y. Xia, Z. Q. Ma, Y. L. Wang, et al, "Enhancement of cooperation in prisoner's dilemma game on weighted lattices," Physica A, vol. 390, 2011, pp. 4602-4609.

[27] C. Shen, J. Lu, L. Shi, "Does coevolution setup promote cooperation in spatial prisoner's dilemma game,” Appl. Math.s Comput., vol. 290, 2016, pp. 201-207.

[28] Q. Q. Song, Z. P. Li, C. H. Fu, et al, "Optional contributions have positive effects for volunteering public goods games," Physical A, vol. 390, 2011, pp. 4236-4243. 